\title{
El ALCA: Libre comercio con Plan de Nación
}

- Francisco Javier Ibisate

\section{Una agenda controversial}

El propósito del presente comentario es mostrar que el esperado éxito del Área de Libre Comercio de las Américas (ALCA) requiere explícitamente un Plan de Nación en cada país. Si reducimos los objetivos del ALCA a exportaciones e inversión extranjera que nos generan más empleos, estaríamos truncando los objetivos mayores del tratado de Quebec. En consecuencia, si esperamos que un área de libre comercio continental, gracias a reducciones arancelarias y de trabas burocráticas, generará por sí misma la expansión de nuestras exportaciones y la entrada de inversión extranjera, seguramente quedaremos bastante desilusionados. He llegado a esta conclusión luego de redactar un breve artículo: El ALCA, un acuerdo entre desacuerdos'.

La firma del ALCA ha generado fuertes controversias y rechazos, porque muchos grupos sociales lo ven como un canal abierto para expandir el libre juego del mercado y de la globalización en el ámbito continental. El rechazo se hizo patente en el Foro Social Mundial de Porto Alegre, celebrado en enero de 2001 y tuvo su eco en la Cumbre de los Pueblos, convocada en Quebec. Bastantes seminarios y documentos muestran que en Latinoamérica, los desequilibrios económicos, sociales y humanos gestados por la globalización superan los efectos positivos que sí se reconocen. Por ello, y a modo de ejemplo, introduzco un resumen del documento El neoliberalismo en América Latina.

Incluso, desde el seno de los Estados Unidos, sindicalistas, ecologistas y sectores del Partido Demócrata, se oponen a autorizar al presidente Bush, igual que lo hicieron con Clinton, la firma de Tratados de Libre Comercio (fast-track) con

\section{1}

El Alca: Libre comercio con Plan de Nación 
otros países, sin una previa revisión del Congreso. Por añadidura, y es importante, para tener éxito en una negociación comercial se requiere la previa capacitación en una serie de exigencias tecnológicas e institucionales, bien detalladas en un trabajo presentado por Carolina Alas, de FusADEs. Sin duda, lo más importante del ALCA son los "anexos" adjuntos a este tratado y que coinciden ampliamente con las ocho iniciativas que acompañan el Plan Puebla-Panamá, recientemente firmado por los presidentes de Mesoamérica. Un elemento crucial en estos tratados es la traducción que se le dé al término democracia y al orden democrático.

Si los gobiernos signatarios han fijado la fecha de inicio del ALCA para el año 2005 significa que les queda un largo camino por recorrer. El mismo punto de partida es controversial, ya que requiere un orden democrático en los países signatarios. En Quebec se ha enfatizado la cláusula democrática, según la cual "toda alteración o interrupción constitucional del orden democrático en un Estado del hemisferio constituye un obstáculo insuperable para participar en la cumbre de las Américas". La controversia girará en torno al concepto de democracia y de orden democrático. Como indica la corresponsal de Le Monde, "con esta cláusula los treinta y cuatro signatarios de las Américas deciden ligar el libre comercio con la democracia"'. Con las mismas fechas, Janette Habel, corresponsal de Le Monde Diplomatique, afirma que el nuevo orden que se quiere imponer se fundamenta en'dos principios: "El principio jurídico de la igualdad de trato y el principio económico de la libre competencia" ${ }^{3}$. Ciertamente se trataría de una integración asimétrica que esterilizaría el fortalecimiento de la democracia.

Es cierto que George W. Bush ha repetido en Quebec: "Creo que el comercio es importante para este hemisferio. No sólo expande la prosperidad, sino que ayuda a expandir la libertad". Bush dijo que quiere construir "un hemisferio democrático, ligado por la buena voluntad y el libre comercio". Pero, desde los "extramuros" de la convención, los representantes de la Cumbre de los Pueblos respondían: "Hacemos una llamada a los pueblos de las Américas para combatir el proyecto ALCA y construir otras alternativas de integración fundadas en la democracia, la justicia social y la protección del medio ambiente"4. Al hablar de un "acuerdo entre desacuerdos", no me refiero sólo a la Cumbre de los Pueblos. El presidente Fernando Cardoso, en su discurso de apertura, condicionó su adhesión al libre comercio exigiendo una "apertura recíproca", criticando las medidas antidumping de los Estados Unidos y las asimetrías existentes en bienes agrícolas. "El ALCA será bienvenido si su creación significa un proceso para dar acceso a los mercados más dinámicos, si efectivamente significa el camino para las reglas compartidas antidumping, si reduce las barreras arancelarias, si evita la distorsión proteccionista de las buenas reglas sanitarias. Pero, si no es así, sería irrelevante o, en el peor de los casos, indeseable"s. El presidente Cardoso criticó también a Bush por no haber querido ratificar el tratado de Kyoto, de 1997, contra el recalentamiento de la atmósfera. Bush dijo que la economía es más importante que la ecología... 
La controversia llega desde otras "altitudes", con exigencias emparentadas con la democracia. En fechas consecutivas, La Prensa Gráfica y El Diario de Hoy repiten la misma noticia: Prevén Congreso no aprobará el fast-track a Bush (11 de mayo de 2001); Senado puede retrasar el last-track. (7 de junio de 2001). Muchos demócratas, con apoyo de los sindicatos, no están de acuerdo en autorizar al presidente la firma de tratados comerciales (fast-track) si no se introducen las cláusulas sobre provisiones ambientales, derechos humanos y laborales. Con estas medidas, dicen, "no están siendo proteccionistas, ni intentan parar el mercado, sino aumentarlo de manera adecuada". Las dudas de los demócratas y sindicatos sobre estos tratados no se refieren sólo al deterioro ecológico, sino a la pérdida de miles de puestos de trabajo en los Eeuu, que ha puesto en tela de juicio el acuerdo comercial con Jordania.

En este escenario de claros desacuerdos, nos preguntamos si la realidad confirmará las expectativas de la declaración de Quebec: "Las economías abiertas y libres, el acceso a los mercados, el flujo sostenido de inversiones, la formación de capitales, la estabilidad financiera, las políticas públicas adecuadas... son claves para reducir la pobreza y la inseguridad, elevar los niveles de vida y promover el desarrollo sostenible... El libre comercio sin subsidios ni prácticas desleales, acompañados de flujos crecientes de inversión productiva y de una mayor integración económica, favorecerá la prosperidad regional, mejorará las condiciones laborales de los pueblos de las Américas y protegerá mejor el medio ambiente". (ECA, 2001, p. 395) De momento, estos son supuestos y expectativas. Por ello, se ha dado un tiempo - hasta 2005- para que los gobiernos, en diálogo con la sociedad, enderecen los caminos hacia este milenario círculo virtuoso del libre comercio mundial. Para ello será necesario iniciar las tareas indicadas en los "dieciocho anexos del tratado", que constituyen un verdadero Plan de Nación. Comencemos con las exigencias básicas que reclama el juego del libre comercio.

\section{El difícil camino del libre comercio}

Tanto el actual como los pasados gobiernos, han querido convertir las exportaciones en uno de los ejes del crecimiento económico. Es claro que se trata de un intento fallido, pese a que el objetivo es bueno y necesario. El multiplicador de comercio internacional tiene, en teoría, sensibles efectos dinamizantes de la economía; la pregunta obvia es por qué esos beneficios no los observamos entre nosotros; incluso, por qué algunos grupos empresariales manifiestan sus temores ante la apertura de nuevos tratados comerciales. Si el eje de las importaciones no ha funcionado, no creo que se trate de una simple deficiencia del gobierno; el problema es, al mismo tiempo, más nacional e internacional. El análisis del difícil camino hacia el libre comercio mundial, sería una de las primeras facetas de nuestro Plan de Nación. Lo necesitamos por razones aritméticas y contables. En la revista del Banco Central, el listado de nuestras importaciones lleva nueve páginas, mientras que el de las exportaciones tan sólo ocupa tres. Esto se tradu-

El alca: Libre comercio con Plan de Nación 
ce en el crónico y creciente déficit de la balanza comercial. Nuestra economía no es generadora de dólares, sino hambrienta de dólares. Lo disimulamos integrando la maquila como si fuera una auténtica industria nacional y maquillando la balanza corriente con el flujo de remesas, que también engordan la Renta Nacional Disponible. Se trata de un problema nacional.

Carolina Alas presenta un ordenado análisis del nuevo entomo internacional del comercio, en los números 179 y 180 del Boletín Económico y Social de FuSADES. En este comentario, me ciño a la segunda parte de su estudio, porque detalla cuáles son los requisitos para insertarse con éxito en la economía mundial. Para comenzar, un dato que tiene importancia: El Salvador se adhirió al Acuerdo General sobre Libre Comercio y Aranceles (GATT), en 1991. Somos miembros relativamente novatos del GATT, convertido en 1995 en la Organización Mundial del Comercio (OMC). En poco tiempo, nuestro gobierno agregó su firma al ALCA y gustaría de verse integrado en un TLC con los Estados Unidos. Desde 1959, somos miembros del Mercado Común Centroamericano, MCCA. Nuestro gobierno tiene puesta su fe en que el libre comercio es germen de prosperidad cconómica.

Alas nos muestra que ese camino requiere serias reformas en el mediano y largo plazo. El comercio internacional está bastante regulado, no sólo en lo referente al intercambio de bienes y servicios, sino al contencioso tema de la inversión, de las normas laborales, ambientales, de la propiedad intelectual y otros temas en litigio desde la cumbre de la OMC, celebrada en Seattle, en 1999. La competencia y la eficiencia son los parámetros del mercado internacional; si el rezago tecnológico, la deficiente infraestructura y la mala calidad de los servicios públicos hacen difícil la reconversión del aparato productivo, "la desintegración que puede generarse con la apertura rápida de fronteras generará desempleo, con los consiguientes conflictos sociales".

\subsection{Proteccionismo y rezago tecnológico}

La autora del análisis que citamos líneas arriba asevera que las economías centroamericanas nacieron y se mantuvieron bajo la sombrilla de una fuerte protección arancelaria. El recordado investigador Salvador Osvaldo Bran lo dice en forma más directa: "Aspecto de singular importancia es cómo superar el patrón de comportamiento tradicional del sector empresarial que cree en el principio del menor esfuerzo tecnológico. El proteccionismo excesivo de varios decenios provocó que éste eludiera correr riesgos, en particular, los asociados a la innovación tecnológica. Muchos empresarios se han acostumbrado a no competir con mejor calidad y precios; prefieren hacerlo con publicidad, acceso a crédito preferencial y la obtención de subsidios del gobierno; o sea, se habituaron a formas de competencia que exigen poco o ningún esfuerzo tecnológico, práctica que debe crradicarse para ser más competitivos" (ECA, 1997: p. 546) 
El reverso de la medalla es que los países socios mantengan una fucrte protección de sus exportaciones, lo que anula las ventajas competitivas de las pequcñas economías. Este problema lo planteó Fernando Cardoso en Quebec y ya fue el epicentro de los disturbios de la reunión de la OMC en Seattle: el tema de la protección del acero es causa de conllicto en el presente mes de junio. Vivimos un libre comercio dentro del océano de la globalización, punto sobre el que volveremos más adelante.

\subsection{Los capitales especulativos}

Alas menciona también las súbitas salidas de capitales golondrinas, que "pueden sumir al país en una grave crisis económica rápidamente”. La crisis lïnanciera de 1997 nació en el continente sudasiático, entre los tigres o dragones que nos presentaban como modelos económicos. El conocido financista y filántropo George Soros — que hizo una fortuna en estos mercados financieros- ha pedido su regulación, porque los grandes desequilibrios nacen de este inestable epicentro. No somos inmunes a estos flujos linancieros porque en este mercado se mezclan aguas negras. En la cumbre de Okinawa, Japón, celebrada en julio de 2000, los miembros del G-7, FMI y BM, "resaltaron los peligros derivados del blanqueo de dinero para la economía mundial y se comprometieron a desarrollar más la persecución a fin de contrarrestar el narcotráfico y el comercio ilegal de armamento. También se comprometieron a vigilar más las irregularidades de los llamados paraisos fiscales". Se presentó una lista de catorce países implicados en estos tráficos —entre ellos, Rusia e Israel-, solicitando una mayor supervisión del sistema bancario, que hace de puente entre el sector real de la economía y las corrientes especulativas del ciberespacio. Hamos vivido la experiencia de Insepro-Finsepro y de anteriores fraudes, que espantan a la inversión extranjera.

Si la empresa privada debe hacer un esfuerzo de reconversión tecnológica, al Estado le incumbe la tarea de invertir en infraestructura y servicios públicos, así como dar signos reales de querer combatir la corrupción, tema presente en los "anexos" de Quebec. No puede haber avances tecnológicos permanentes si no cxisten programas e instituciones dedicadas a la capacitación personal en los distintos niveles, desde la formación escolar hasta la diversificación aplicada de los estudios superiores universitarios. A este propósito, valga hacer dos observaciones. El gobierno hace énfasis en la creación de infocentros en el territorio nacional, programa plausible, por cuanto ayuda a descubrir y orientar las corrientes del mercadeo. Pero los infocentros deben presuponer la existencia de escuelas profesionales diversificadas que capacitan la mano de obra según las necesidades empresariales presentes y futuras. En El Salvador tenemos un excedente de universidades que preparan, mejor o peor, profesionales de "cuello blanco", al mismo tiempo que existe un amplio déficit de escuelas tecnológicas que especialicen las "uñas azules" que demandan las empresas. En las encuestas de FUSADES, la carencia de personal calificado aparece como una de las quejas

El alca: Libre comercio con Plan de Noción 
empresariales. No basta decir que tenemos una mano de obra laboriosa si se queda en sólo mano de obra.

Como la capacitación de mano de obra aparece como una recomendación especial en los anexos de Quebec, otra recomendación repetida por la Comisión Nacional de Ciencia y Tecnología (CONACYT) es la mayor integración entre el sector productivo y en las universidades con carreras de ingeniería y ciencias empresariales, en un proceso de retroalimentación. Cuando en la pasada década participamos en la gestación de la Ley de Educación Superior, sugerimos que en el Consejo Superior de Educación estuviera presente un miembro del Ministerio de Planificación que orientara a las universidades para abrir aquellas carreras más necesarias para el presente y futuro próximo del país. La sugerencia quedó en el tintero, porque el gobierno de Calderón Sol (léase Manuel E. Hinds) derruyó dicha institución, y todo quedó en manos del libre albedrío, es decir, más de lo mismo. La integración del sector productivo y de los centros de investigación, así como la capacitación profesional de la mano de obra, son un doble trampolín hacia el mercado internacional.

\subsection{El apoyo financiero internacional}

De esta forma, Carolina Alas va apuntando o apuntalando una serie de soportes necesarios para embarcarnos en el océano global. Un soporte necesario para la reconversión industrial será "el apoyo financiero de organismos internacionales y de las potencias económicas, que puedan hacer más fácil la adquisición de nueva tecnología". En este punto, nos enfrentamos al dilema de la necesidad de este apoyo y de la respuesta internacional. En la cumbre de Bangkok, Tailandia, de febrero de 2000, epicentro de la crisis financiera de 1997, Rubens Ricupero, secrelario de la UNCTAD, resume su discurso en esta expresión: "La globalización hace difícil la globalización". "Los pobres se benefician relativamentc poco de la afluencia de capitales, pero cargan gran parte del costo cuando se realiza la fuga de capitales y se deterioran los servicios sociales. Por otra parte, la distribución geográfica de la inversión extranjera ha sido muy desigual y concentrada en un pequeño número de países. Sólo los gobiernos que disponen de medios necesarios pucden dedicar fondos a la investigación y promoción de nucvas tecnologías y de la competitividad de productos transables. Las nucvas tccnologías son ascguradas por "derechos de propiedad intelectual", derechos onerosos para los países pobres e imposibles para los más pobres" (Realidad, 1999, pp. 664-668). El respeto a la propiedad intelectual es uno de los "anexos" aceptado por los signatarios del ALCA.

Carolina Alas recucrda que este proceso de reconversión y modernización es "altamente dinámico y cvolutivo". A ello se agrcgan otros signos de economía saludable: "la estabilidad macrocconómica, estabilidad de precios, disciplina fiscal, deuda extcrna prudentc, estabilidad del tipo de cambio, políticas orientadas 
a la apertura externa y a los mecanismos de mercado y funcionamiento de la democracia". Para algunos gobiernos signatarios del ALCA, habría una correlación significativa entre apertura externa, mecanismos de mercado y funcionamiento de la democracia. Esta teoría es hoy día un tanto controversial y debe confrontarse con la realidad. Dijimos antes que los representantes de la Cumbre de los Pueblos of recen cambiar el eje "comercio para la democracia representativa" por el eje "democracia participativa para el comercio". (ECA, 2001; p. 395-399). Es necesario ir integrando el ALCA en el marco y en el juego de la globalización.

\subsection{Estrategias de negociación}

La autora del análisis detalla "las exigencias (voluntarias o impuestas) para poder ser aceptado como socio potencial y poder negociar acuerdos, sobre todo, con países más desarrollados". El primer requisito sería "una estrategia de negociación sólida", que comentaremos más adelante. Se agregan una serie de requisitos obligatorios, algunos derivados de las normas de la OMC; en Seattle salió a la luz que los primeros en incumplirlos son las trilaterales de Estados Unidos, la Unión Europea y Japón. Otros se derivan del acuerdo bilateral o regional, tal es el caso del ALCA. Nuestro análisis se centra en el ALCA y, puesto que George W. Bush hijo es el "padre del ALCA", es obvio que se impondrán algunos principios comerciales propios de los Estados Unidos. Los europeos piensan que el gobierno de Estados Unidos está muy interesado en que se apruebe el acuerdo del ALCA, que integraría 800 millones de personas de treinta y cuatro países signatarios. Como la OMC no se ha vuelto a reunir desde 1999, las cláusulas del acuerdo del ALCA pudieran transformarse en la normativa actualizada de la OMC en Ginebra. Así se está actualizando la obra de Lester Thurow: La guerra del siglo xxI (head to head): La batalla económica entre Japón, Europa y los Estados Unidos.

En este escenario se entienden mejor ciertos requisitos exigidos por los Estados Unidos para ser socios de la OMC: "La integración estará basada en la reciprocidad y no esperar ningún trato diferenciado con base en su desigual desarrollo". Cumplir con las normas laborales y del medio ambiente; "Haber realizado importantes avances en la liberalización del comercio y proporcionar un trato justo y equilibrado a las importaciones de los Estados Unidos. Se le da gran importancia a la protección de la propiedad intelectual, el trato nacional que se otorga a la inversión extranjera, la eliminación de obstáculos al comercio de servicios y el manejo de subsidios a las exportaciones"... El Salvador ha llevado a cabo un "agresivo programa de desgravación" a la propiedad intelectual... y "se ha otorgado mayores garantías al inversionista extranjero en cuanto a la repatriación de utilidades, posesión de bienes y competencia leal"... Al mismo tiempo, Carolina Alas recuerda el rezago tecnológico como una debilidad común a las pequeñas economías. 


\subsection{La calificación de la CEPAL}

La Comisión Económica para América Latina (CEPAL) ha analizado el grado de preparación de los países pequeños para insertarse con éxito en la economía mundial. Se cuantifica con bastante detalle los requisitos de eligibilidad, el grado de preparación estructural y el grado de preparación de las políticas y de riesgo. De acuerdo con estos parámetros, la CEPAL calificó a los países en muy alto, alto, regular y bajo. Según estos criterios, "la elegibilidad macroeconómica de El Salvador era baja, la preparación estructural económica y sectorial era regular, la diversificación y dinamismo exportador muy alto, el recurso humano bajo, la política macroeconómica baja, la política comercial alta, la política de apoyo a la transformación productiva baja, la vulnerabilidad comercial regular y la vulnerabilidad macroeconómica muy alta". Contrastan un tanto estas mediocres calificaciones con las expectativas optimistas de nuestro gobierno al firmar el acuerdo del ALCA y, en su defecto, un tratado de libre comercio con los Estados Unidos. Bajo cualquier alternativa, y bajo la hipótesis de que el libre comercio mundial genere efectos tan contundentes, la inserción en estos tratados requiere un plan nacional concertado con la sociedad. Esta es una de las recomendaciones más repetida en Quebec.

Por añadidura, Carolina Alas nos recuerda que aún tenemos tangibles debilidades en la estrategia de negociación. Nuestra adhesión a la OMC es relativamente reciente - 1991 - y el equipo negociador - Ministro de Economía - ha ido ganando cierta experiencia en el TLC con México. Los miembros del primer equipo negociador se retiraron y los miembros de la segunda generación han podido constatar que los delegados mexicanos nos llevan una gran delantera luego de sus negociaciones con Canadá y los Estados Unidos. Cuentan con sólidas instituciones asesoras y promotoras del mercadeo externo, así como con una economía más diversificada. Este es un nuevo reto y constituye un nuevo aprendizaje. "Esta estrategia de negociación debe, en primer lugar, considerar que, pese a la desigual condición económica que puedan tener los miembros del bloque, no podrán exigirse concesiones unilaterales, sino que debe esperarse la reciprocidad en el trato, aunque sí podrán negociarse los plazos y condiciones en que se llegará al cumplimiento total de los compromisos y las normas de origen. Obviamente, el éxito de una está directamente vinculado con la capacidad y experiencia del equipo negociador". Luego de detallar estos puntos de la negociación, hace una advertencia digna de la mayor atención: "La participación en los bloques regionales no significa que será un socio exitoso, a menos que se negocie en forma conveniente su entrada y se prepare a la economía para enfrentar los desafíos que implica su globalización"

Con esta última advertencia, Carolina Alas nos sugiere, sobre todo al gobierno, reflexionar sobre los siguiente: Que tenemos todavía serias deficiencias y restricciones para penetrar con éxito en la economía internacional; las deficien- 
cias concretas han sido claramente puntualizadas, a sabiendas de que el "eje exportación" puede ejercer un fuerte efecto multiplicador. Pero, tanto o más importante es preguntarnos si nuestra cconomía está preparada para enfrentar "los desafíos que implica la globalización". En la segunda Cumbre de las Américas, llevada a cabo en Santiago de Chile en abril de 1998, se planteaba el problema: "La globalización ofrece grandes oportunidades para el progreso de nuestros países y abre nuevos campos de cooperación para la comunidad hemisférica. Sin embargo, puede también incidir en un aumento de las diferencias entre los países y al interior de nuestras sociedades... La superación de la pobreza sigue siendo el reto más grande al que se enfrenta nuestro hemisferio. Estamos conscientes de que el crecimiento positivo observado en las Américas en los últimos años no ha solucionado todavía los problemas de inequidad y exclusión" ( $E l$ Diario de Hoy, 22 de abril de 1998; pp. 64-65). Parece que algunos de los gobiernos signatarios de Quebec ya no recuerdan esta advertencia de sus predecesores en Chile.

Se hace necesario descubrir las relaciones asimétricas que imperan en el mercado de la globalización, donde las grandes potencias controlan a las mismas instituciones internacionales (FMI, BM, OMC...) doblegando, a su favor, la teoría de las ventajas competitivas. Desde la Cumbre sobre el Desarrollo Social, efectuada en Copenhague en 1995, este ha sido uno de los puntos de litigio en los foros internacionales celebrados de 1999 al 2001. La economía mundial y la teoría del libre comercio adquiere nuevas relaciones asimétricas bajo la égida de la globalización. He tratado de presentar estas posiciones divergentes en el artículo ya citado: El ALCA: Un acuerdo entre desacuerdos. Superabunda la bibliografía sobre el mismo tema, por lo que me ciño a recomendar la obra de Edward Luttwak: Turbocapitalismo, por ser un actualizado análisis de la geoeconomía?.

La conclusión no es cerrarse a la apertura comercial, y más bien se está llegando a la teoría de la concertación: puesto que la globalización es imparable, se hace necesario "gobernar la globalización". Luego de la crisis financiera de 1997, el Grupo de los 7, FMI y BM, han recomendado aplicar medidas más severas de supervisión y control de los sistemas bancarios y de los Estados, incluidos los paraísos fiscales, que facilitaron la volatilidad de los capitales especulativos bancarios. Pero no es éste el único ingrediente de la globalización. Llama la atención que en el tratado del ALCA se incluyan dieciocho anexos, no propiamente comerciales, que sirven para preparar y proteger a los pequeños países signatarios contra las situaciones asimétricas económicas y sociales existentes en el desigual continente panamericano. Si en el ámbito mundial hemos entrado en la "cra de los manifestantes". es normal que este fenómeno lo observamos a escala latinoamericana. A modo de prefacio a esos anexos y, sobre todo, en orden a apreciar objetivamente los beneficios y los desajustes generados por la globalización, podemos resumir un documento preparado desde el universo de la América Latina y que ha sido discutido en posteriores seminarios. 


\section{El neoliberalismo en América Latina}

Dieciocho supcriores provinciales de la Compañía de Jesús en América Latina lïman, en noviembre de 1996, un documento preparado por un equipo de prolesionales. En la introducción, se dice que se trata de "algunas reflexiones sobre el llamado neoliberalismo en nucstros países. Nuestra prcocupación principal es de orden ético y religioso... Nos resistimos a aceptar tranquilamente que las medidas económicas aplicadas en los últimos años, en todos los países latinoamericanos y del Caribe, sean la única manera posible de orientar la economía, y que el empobrecimiento de millones de latinoamericanos sea un costo irremediable de un futuro crecimiento". A continuación, presento un resumen (lel documento El neoliberalismo en América Latina.

\subsection{La sociedad de que somos parte}

En cl umbral del siglo XXI, las comunicaciones nos unen estrechamente, la tccnología nos da nucvas posibilidades de conocimiento y de creatividad, y los mercados penetran todos los espacios sociales. En contraste con la década pasada. la economía de la mayoría de los países latinoamericanos ha vuelto a crecer. Sin cmbargo, cste auge material, que podría abrir esperanzas para todos, deja multitudes en la pobreza, sin posibilidad de participar en la construcción del destino común, amenaza la identidad cultural de nuestros pueblos y destruye los recursos naturales. Calculamos que en Latinoamérica y en el Caribe por lo menos 180 millones de personas viven en la pobreza y 80 millones sobreviven en la miseria.

\subsection{Las políticas económicas neoliberales}

Ellas ponen el crecimiento económico - y no la totalidad de los hombres y mujeres en armonía con la creación- como razón de ser de la economía. Restringen la intervención del Estado hasta despojarlo de responsabilidades sobre los bienes mínimos que merece todo ciudadano, por ser persona. Eliminan los programas generales de oportunidades para todos y los sustituyen por apoyos ocasionales a favor de grupos particulares. Privatizan empresas con el criterio de que en todos los casos el Estado es un mal administrador. Abren sin restricciones las fronteras a mercancías, capitales y flujos financieros y dejan sin suficientc protección a los productores más pequeños y débiles. Pasan en silencio el problema de la deuda externa, cuyo pago obliga a recortar drásticamente la inversión social. Subordinan la complejidad de la hacienda pública al ajuste de las variables macroeconómicas: presupuesto fiscal equilibrado, reducción de la inflación y balanza de pagos estables, como si de allí se siguiera todo bien común y no se generaran nuevos problemas para la población.

Insisten en que estos ajustes producirán un crecimiento que, cuando sea voluminoso, elevará los niveles de ingreso y resolverá, en consecuencia, la situación de los desfavorecidos. Eliminan los obstáculos que podrían imponer las 
legislaciones que protegen a los obreros, para incentivar la inversión privada. Liberan de impuestos y de las obligaciones con el medio ambiente a grupos cconómicamente fuertes, y los protegen para acelerar el proceso de industrialización: con ello provocan una concentración todavía mayor de la riqueza y el poder cconómico. Ponen al servicio de esta estrategia económica la actividad política, al quitar toda traba, todo control político y social para lograr la hegemonía del mercado libre en todo campo, incluso en la contratación de la mano de obra.

\subsection{Sus efectos}

Reconocemos que las medidas de ajuste han tenido también aportes positivos. Los mecanismos de mercado han elevado la of erta de bienes de mejor calidad y precios. La inflación se ha reducido en todo el continente. Los gobiernos han dejado tareas que no les competen para dedicarse, como es su deber, al bien común. Se ha generalizado la conciencia del valor de la austeridad fiscal que utiliza mejor los recursos físicos. Y las relaciones comerciales entre nuestras naciones han logrado un avance significativo.

Estos elementos, sin embargo, están lejos de compensar los desequilibrios generados: gran concentración de los ingresos, la riqueza y la propiedad de la tierra; multiplicación de masas urbanas sin trabajo o que subsisten en empleos inestables y poco productivos; quiebras de miles de pequeñas y medianas empresas; destrucción y desplazamiento forzado de poblaciones indígenas y campesinas; expansión del narcotráfico basado en sectores rurales, cuyos productos tradicionales quedan fuera de competencia; desaparición de la seguridad alimentaria; aumento de la criminalidad, provocada no pocas veces por el hambre, desestabilización de las economías nacionales por los flujos libres de la especulación internacional, desajustes en comunidades locales por proyectos de empresas multinacionales que prescinden de los pobladores.

En consecuencia, al lado de un crecimiento económico moderado aumenta, en casi todos nuestros países, el malestar social que se expresa en protestas ciudadanas. Vuelve a tomar fuerza, en algunos lugares, la lucha armada, que nada soluciona. Aumenta el rechazo a la orientación económica general que, lejos de mejorar el bien común, profundiza las causas tradicionales del descontento popular: la desigualdad, la miseria y la corrupción.

\subsection{La concepción del ser humano}

Detrás de la racionalidad económica "neoliberal", hay una concepción del ser humano que delimita la grandeza del hombre y la mujer a la capacidad de generar ingresos monetarios. Esto exacerba el individualismo y el afán de ganar y poscer, y lleva fácilmente a atentar contra la integridad de la creación. En muchos casos desata la codicia, la corrupción y la violencia. Así, al generalizarse en los grupos sociales, destruye radicalmente la comunidad. Se impone, por

El alCA: Libre comercio con Plan de Nación 
tanto, un orden de valores en donde prevalece la libertad individual para acceder al consumo en las satisfacciones y placeres; legitimando, entre otras cosas, la droga y el erotismo sin restricciones. Una libertad que rechaza cualquier interferencia del Estado en la iniciativa privada, que desconoce la virtud de la solidaridad y que sólo acepta las leyes del mercado.

Por el proceso de globalización de la economía, esta manera de comprender al hombre y la mujer penetra nuestros países con unos contenidos simbólicos de gran capacidad de seducción. Gracias al dominio que ejerce esta visión sobre los medios de comunicación de masas, se rompe la identidad de culturas locales que no tienen voz para hacerse oír. Los dirigentes de nuestras sociedades, normalmente articulados con estos movimientos de globalización y embebidos en la aceptación indiscriminada de las razones del mercado, viven como extranjeros en sus propios países. Sin dialogar con el pueblo, lo consideran obstáculo y peligro para sus intereses, y no como hermano, compañero o socio... En consecuencia, al mirar la región en conjunto, se descubre que las políticas neoliberales profundizan problemas estructurales que están en la base de la pobreza: la distribución de la riqueza, el capital social y las distorsiones sociales generadas por el mercado cuando actúa sin control ${ }^{R "}$. Hasta aquí la síntesis del documento.

Cuatro meses después, Franz Hinkelammert introduce así su artículo sobre El huracán de la globalización: "El proceso de la globalización pasa desde hace más de dos décadas por encima de América Latina, así como pasa por encima del mundo entero, como un huracán. La privatización de las funciones del Estado, el libre comercio, el desencadenamiento de los movimientos internacionales de los capitales, la disolución del Estado Social, la entrega de las funciones de planificación económica a las empresas multinacionales y la entrega de la fuerza de trabajo y de la naturaleza a las fuerzas del mercado, han arrollado el continente"'.

\section{Los anexos no comerciales del ALCA}

Con fecha 2 de abril, El Diario de Hoy presenta el escudo y los principios del ALCA: Preservar y fortalecer la comunidad de democracias de las Américas. Promover la prosperidad a través de la integración económica y el libre comercio. Erradicar la pobreza y la discriminación en nuestro hemisferio. Garantizar el desarrollo sostenible y conservar nuestro medio ambiente para las generaciones futuras". En la edición del 23 de abril de ese matutino, se enuncian los dieciocho compromisos anexos al documento oficial: 1) Democracia; 2) Derechos humanos; 3) Justicia; 4) Seguridad; 5) Sociedad civil; 6) Comercio inversión; 7) Infraestructura; 8) Cooperación ante desastres naturales; 9) Medio ambiente; 10) Gestión agrícola y desarrollo rural; 11) Trabajo y empleo; 12) Crecimiento con equidad; 13) Educación; 14) Salud; 15) Igualdad de género; 16) Pueblos indígenas; 17) Diversidad cultural; 18) Infancia y juventud. Ante este tablero de ajedrez, la pregunta obvia es: ¿quién es el ALCA, quiénes los anexos 
y cuál la relación entre el ALCA y los anexos? Nos da la impresión de estar frente a un Plan de Nación para cada país.

\subsection{Los anexos del ALCA}

Con mayor detalle, вBC Mundo presenta estos principios y anexos. El objetivo central es que el Área de Libre Comercio de las Américas sirva para la reducción de la pobreza y el fortalecimiento de la democracia. A este fin se agregan otra serie de objetivos, nada fáciles de obtener a largo plazo. "La transparencia de las relaciones entre las instituciones y los ciudadanos, dándosele mayor importancia a la sociedad civil. La cooperación en materia de derechos humanos, la promoción de los derechos civiles, económicos, políticos y sociales de la mujer y de los pueblos indígenas. El acceso universal a sistemas de justicia imparciales e independientes, y la necesidad de recurrir a éstos para resolver conflictos. La creación de prosperidad con la mayor zona de libre comercio del mundo -800 millones de personas-, impulsar los principios de inclusión y equidad, así como mejorar las condiciones de trabajo, el respeto a las normas laborales y la cooperación en las cuestiones relacionadas con la migración.

En este marco de la globalización, hay que estudiar la adopción de iniciativas destinadas a garantizar la buena gestión de las empresas privadas y su responsabilidad social. Mejorar los sistemas de transporte en el hemisferio, enfoques comunes en cuanto a la energía y estrechar la cooperación en cuestiones medioambientales. La realización del potencial humano, que permita mejorar el nivel de vida de todos los ciudadanos, apoyando la iniciativa de reducir a la mitad, antes del 2015, el número de personas que viven en la pobreza en todo el continente. Potenciar el acceso al empleo y facilitar que los ciudadanos adquieran las aptitudes necesarias para competir en el contexto de una economía mundial basada en el conocimiento. Encontrar soluciones prácticas a la "brecha digital" existente en el hemisferio. Énfasis en el acceso a la educación, la asistencia médica, así como proteger y promover la diversidad cultural"10.

\subsection{Un Plan de Nación}

Como el listado de los anexos ha sido transcrito literalmente de BBC Mundo, sin duda tenemos la impresión de estar frente a un aluvión de objetivos dispersos. Incluso, tratando de agrupar los temas, estamos delante de un denso curso de Problemas Económico-Políticos Continentales. ¿Qué es lo que nos quieren comunicar los gobiernos signatarios con estos principios y estos anexos? Después de haber leído el documento El neoliberalismo en América Latina, estamos de acuerdo en la tarea de corregir y de avanzar en cada uno de los dieciocho anexos. Lo que no aparece tan claro es que el libre comercio mundial ayude a la floración espontánea de estos anexos; más bien, pasa de largo frente a ellos. Los signatarios de Quebec nos vienen a decir que es necesario "gobernar la globa-

El alca: Libre comercio con Plan de Nación 
lización" mediante una acción concertada del Estado y la sociedad civil. Si los anexos no son un simple adorno para justificar el tratado del ALCA, lo que tenemos aquí es un esquema de relorma del modelo neoliberal. Pero si los anexos son un juego de fuegos artificiales para cclebrar la firma del ALCA, todo terminará en la mayor desilusión y en el rechazo de muchas cumbres de los pueblos.

\subsection{Reducir la pobreza y fortalecer la democracia}

No es posible analizar todos los anexos y bastan algunos comentarios. Se dice que el ALCA debe servir a la reducción de la pobreza y al fortalecimiento de la democracia. Este es un objetivo tan laudable como dilícil. En el ámbito mundial, el libre comercio internacional, regulado por la $\mathrm{OMC}$-o por quienes regulan a la $\mathrm{OMC}$ - ha logrado más bien efectos contrarios. Ofrezco sólo tres documentos internacionales. La cumbre mundial de Copenhague, de 1995, prescnta, en sus documentos de antecedentes, tres problemas mundiales: se desarrolla la pobreza, el crecimiento con desempleo y la atomización e insolidaridad social. (Realidad, $\mathrm{N}^{\circ}$ 54, nov.-dic. 1996). James Wolfensohn, Presidente del Banco Mundial, pronuncia, ante los gobernadores de dicha institución, su discurso sobre La otra crisis, en oclubre de 1998: “... Hoy, mientras hablamos de crisis lïnancicra, en todo cl mundo, I.300 millones de personas subsisten con menos de un dólar al día; 3.000 millones viven con menos de dos dólares al día; I.300 millones no lienen agua potable; 3.000 millones carecen de servicios de saneamiento y 2.000 millones no tienen electricidad". El discurso entero puede leerse en ECA, 1998 (pp. 1003-1009). No significa esto que la pobreza nació con la globalización, sino que la globalización ha profundizado la pobreza tradicional, como lo dice el siguiente documento.

En el balneario suizo de Davos sc reúne cada año la élite del capitalismo. La agenda de Davos-1999 era: La globalidad responsable: la gestión del impacto de la globalización. Los documentos de antecedentes, presentados por los organizadores del foro, adelantan duras crílicas a estc proceso. "La globalización debe adquirir un compromiso social que no tenga como contrapartida la miseria y la exclusión de millones de seres humanos en el planeta. Esta crisis es el resultado de una globalización que ha sido conducida de manera irresponsable. Los problemas crcados por la mundialización han desembocado en una crisis sistémica. O se diseñan nuevas medidas para hacer frente a la crisis, o estamos condenados a entrar en un período de caos endémico y sistemático"... "Miseria y desempleo, destrucción masiva de la riqueza financiera y material. Estados sometidos a la especulación, un capitalismo salvaje que erosiona las normas más elementales de la convivencia, son algunos de los rasgos que advertirían una globalización irresponsable. De ahí la necesidad de que el fenómeno recupere su rostro humano"... (Cfr. Apuntes de Sistemas Económicos, p. 235)

El lcgado del representante del Banco Mundial, en su reciente visita a El Salvador, ha sido: "disminuir la pobreza, primer reto". Los terremolos han ele- 
vado el nivel de pobreza por encima del $50 \%$ de la población nacional, porcentaje ya existente antes del cataclismo. Esto significa que, sólo en el muy largo plazo, el libre comercio internacional pudiera, talvez, aliviar un problema tan estructural y que es necesario aplicar medidas directamente dirigidas a aliviar la pobreza y fortalecer la democracia. En concreto, el Banco Mundial propone una agenda social: "Educación básica y secundaria a nivel nacional. Atención de salud pública para aumentar la cobertura. Desarrollo rural. Infraestructura básica en zona rural —caminos y electricidad-. Modernización del Estado, gobernabilidad y reforma judicial. Mejoramiento de los barrios marginales urbanos. Manejo de recursos naturales y medio ambiente. Descentralización y desarrollo local" (Cfr. El Diario de Hoy, 19 de abril de 2001, p. 28). Esta agenda social es parte integrante de los anexos del ALCA, confirmando que estos objetivos no son un efecto, sino un requisito previo a la apertura del libre comercio internacional.

En los documentos gubernamentales y en el discurso presidencial del $1^{\circ} \mathrm{de}$ junio, sc espera que el ICC con los Estados Unidos, el TLC con México y otros tratados comerciales, generen una secuencia de miles y miles de nuevos empleos. Ojalá así sea. Pero esto vendría simplemente a reponer la pérdida de otros miles de empleos generados por la destrucción de tantas micro y pequeñas empresas; además, la misma apertura comercial puede dejar en situación difícil a otras empresas poco competitivas y elevar así la tasa de situación difícil a otras empresas poco competitivas y elevar así la lasa de desempleo, como lo han expresado algunos sectores empresariales. No podemos esperar que el problema del desempleo y pobreza estructural se solucione "desde afuera", cuando tienen otras raíces internas, entre ellas, la concentración de la riqueza generada por el mismo modelo de libre mercado. La reducción de la pobreza requiere otras políticas económicas y reformas estructurales ya indicadas en recientes documentos: Bases para un Plan de Nación, Temas claves para un Plan de Nación... Es importante que en la agenda de Quebec se afirme que el fortalecimiento de la democracia requiere la reducción de la pobreza. Uno de los enfrentamientos es si hablamos de la democracia representativa o de la democracia participativa.

\subsection{El diálogo con la sociedad civil}

Un avance hacia la democracia representativa es la petición fiormulada en Qucbec: "La transparencia de las relaciones entre las instituciones y los ciudadanos, dándosele mayor participación a la sociedad civil". Comenzando por un clato concreto, el Presidente Bush insistió sobre cl carácler democrático de la cumbre: "Lucgo de la reunión, presentarcmos cl acucrdo, o su marco, a todos para que puedan estudiarlo y para que los ciudadanos de todos los países tengan la posibilidad - por primera vez en la historia- de examinar cl texto de un acucrdo comcrcial". Sumando la alïrmación de Bush con la letra del ancxo citado, nos evitaríamos hacer una lectura parcial y no democrática de la llamada "cláusula democrática": "Cualquier alteración o ruplura constilucional del orden

El alca: Libre comerciocon Plan de Nación 
democrático constituye un obstáculo insuperable para la participación del gobierno de dicho Estado en el proceso de la Cumbre de las Américas". Esta cláusula tuvo una dedicatoria concreta (Cuba), y el presidente Francisco Flores ha pedido se incluya también en el Plan Puebla-Panamá, previendo quizás posibles resultados en algunas próximas elecciones dentro del istmo o rumores de golpe de Estado en otro país.

Este sería sólo un aspecto de la incipiente democracia, cuando en muchos países se ha perdido la confianza y credibilidad en las instituciones públicas. Cuántas veces hemos escuchado decir que tenemos libertad de votar, pero no tenemos libertad de elegir, lo cual deriva en el creciente absentismo electoral. No suelen abrirse vasos comunicantes entre las necesidades y demandas de la población y los gobiernos e instituciones públicas, mal calificados en las encuestas de opinión pública. Esta ha sido una de las críticas del Foro Social Mundial de Porto Alegre ("Otro mundo es posible") y de la Cumbre de los Pueblos en Quebec ("Otra América es posible"). El documento firmado por los superiores provinciales de Latinoamérica lo expresa en forma más cruda: "Los dirigentes de nuestras sociedades, normalmente articulados con estos movimientos de la globalización y embebidos en la aceptación indiscriminada de las razones del mercado, viven como extranjeros en sus propios países. Sin dialogar con el pueblo, lo consideran obstáculo y peligro para sus propios intereses, y no como hermano, compañero o socio". Parece ser éste problema continental; de ahí la razón de este primer anexo.

\subsection{No somos la excepción}

El Salvador no escapa a esta crítica, porque obedecemos a instancias mayores. El Dr. Álvaro Magaña - expresidente constitucional y reconocido economista, fallecido recientemente- escribió un artículo sobre La políícica económica de la década de los noventa: necesidad de su revisión. En su primera página, dice: "Así comenzó la que nosotros llamamos la década perdida del neoliberalismo, cuando se inician las políticas que para la globalización exigían el FMI / BM, el binomio cuyo daño que nos ha hecho tuvo un alto precio para la generalidad y que los obedientes líderes de la derecha, ya en el poder, aceptaron y hasia ayudaron para lograr la aprobación de políticas, que ya es hora de revisar, pues estamos bastante retrasados en evaluarlas seriamente y modificarlas para el bien del país". El Dr. Álvaro Magaña tradujo muy bien la sensación, que, incluso tantos académicos han sentido, de estar supeditados los gobiernos a los programas de estas instituciones y de quienes las controlan de acuerdo a la hegemonía del mercado. Así se suprimió el Ministerio de Planificación Económica y Desarrollo Social, y también claudicó el Foro de Concertación Económica y Social, un foro importante, porque en los Acuerdos de Paz había quedado en espera la revisión del modelo económico imperante. 
Desde entonces, ¡cuántos "golpes de timón" improvisados y no consultados!, como lo afirma el recordado investigador Salvador Osvaldo Bran, pues al inicio del segundo gobierno de ARENA se dijo que El Salvador se convertiría en un gran país maquilador; a los pocos meses nadie habló de eso y se comenzó a promover la dolarización de la economía, proyecto que fue abandonado sin conocer las explicaciones; luego se dispuso liquidar la planificación global y enfatizar las políticas sectoriales. Esta acción fue neutralizada y el propio Ministro de Hacienda, en forma insólita, declaró que las políticas sectoriales estaban "pasadas de moda" y que no era productivo promoverlas. Así se llegó a la novedad de impulsar los clusters, o sea, alianzas competitivas, que la gran mayoría de productores desconocía" (Cfr. ECA, 1997, pp. 538-539). Sí se dieron dos golpes de timón, poco transparentes y muy cuestionados: el proceso de privatizaciones y el alza del IVA al 13\%. En este apartado nos estamos refiriendo sólo a los imprevistos económicos como un ejemplo más del cortocircuito entre gobierno central y sociedad civil.

El pasado 22 de noviembre, fuimos testigos pacientes de una determinación inconsulta y precipitada, al presentar la ley de "desintegración monetaria", acompañada de la forma tan turbia en que fue aprobada por la Asamblea Legislativa. Se ha hablado de turbodolarización. Prescindiendo de las bondades y perjuicios de esta ley, el proceso ha sido calificado como dictatorial. Una vez más, el gobierno ha dado muestras de su centralismo en todo el proceso de preparación y presentación del plan de ayuda (Informe-Madrid) ante los países donantes. En esa oportunidad, varias instituciones, como FUSADES, el BID y la CEPAL, pidieron al gobierno abrirse a la concertación con otras instituciones locales y grupos políticos, para dar una mejor imagen de unida nacional. También en todo el proceso de distribución de la ayuda recibida ha primado la polarización política sobre la concertación. En resumen, estamos lejos de la primera recomendación: "La transparencia de las relaciones entre las instituciones y los ciudadanos, clándosele mayor participación a la sociedad civil".

\subsection{El capital humano y la infraestructura productiva}

Coincidiendo en varios puntos con el Plan Puebla-Panamá, los anexos del ALCA enumeran otra serie de requisitos necesarios para ser socios exitosos en cualquier clase de tratado comercial. "Potenciar el acceso al empleo y facilitar que los ciudadanos adquieran las actitudes necesarias para competir en el conlexto de una economía mundial basada en el conocimiento. Encontrar soluciones prácticas a la "brecha digital" existente en el hemisferio. Énfasis en el acceso a la educación, la asistencia médica, así como proteger y promover la diversidad cultural".

Muy en cercanía con las ocho iniciativas del PPP, se requieren avances en la misma infraestructura física productiva. "Mejorar los sistemas de transporte en 
el hemisferio, enfoques comunes en cuanto a la energía y estrechar la cooperación en cuestiones medioambientales". Entre las iniciativas del PPP, la primera se refiere al desarrollo sostenible, preservando el medio ambiente y los recursos naturales de la región; la segunda, recomienda la prevención y mitigación de los desastres naturales; la quinta habla de la integración regional de la infraestructura vial; la sexta propone la conexión energética; la séptima, la integración de los servicios de telecomunicación y la octava, centrada en el desarrollo humano, involucra procesos de educación, salud, desarrollo indígena y cuestiones migratorias.

Se integraría aquí un anexo, transcrito literalmente de la pasada Cumbre del Milenio: "La realización del potencial humano, que permita mejorar el nivel de vida de todos los ciudadanos, apoyando la iniciativa de reducir a la mitad, antes del 2015 , el número de personas que viven en la pobreza en todo el continente". No es el libre comercio el que genera estos efectos, sino que estos efectos son requisitos previos para el éxito deseado en el libre comercio.

\subsection{Los derechos humanos y la justicia}

Es interesante que en la Cumbre de la Organización Mundial del Comercio, efectuada en Seattle, se debatieran algunas de las siguientes exigencias que deben tomar en cuenta cl comercio en el escenario de la globalización. Son exigencias reclamadas por el foro social de Porto Alegre y por la Cumbre de los Pucblos y, de hecho, así aparecen aprobados en Quebec. "La cooperación en materia de dercchos humanos, la promoción de los derechos civiles, cconómicos, políticos y sociales de la mujer y de los pueblos indígenas. El acceso universal a sistema de justicia imparciales e independientes y la necesidad de recurrir a éstos para resolver conflictos. Impulsar los principios de inclusión y equidad, así como mejorar las condiciones de trabajo, el respeto de las normas lahorales y la cooperación en las cuestiones relacionadas con la migración”. Se agrega el componente de la seguridad ciudadana.

Auncjue no existiera el comercio internacional, la promoción y defensa de cstos derechos es parte de lo que entendemos por Estado de Derecho, donde no basta decir cjue todos somos iguales ante la ley; lo importante es que se logre una igualdad real dentro de la sociedad. Lo que sucede es que el libre comercio o lihre mercado internacional arrasa con algunos de estos derechos por el mismo proceso de exclusión. Por este motivo, merece especial atención el siguiente ancxo: "En este marco de la globalización, hay que estudiar la adopción de iniciarincas destinadas a garantizar la buena gestión de las empresas privadas y. su responsabilidad social". Talvez, sin pretenderlo, el acuerdo del ALCA-Quebec recoge un clamor repelido en tantas cumbres mundiales.

Por ello, a modo de resumen, traslado unas rellexiones de Manuel Castells, de la Universidad de Berkeley, lucgo de la cumbre de la OMC en Seattle: "Los mercados hacen unas cosas bien (como asignar recursos escasos y asegurar la 
sclección mediante la competitividad) y otras mal (igualdad social) o muy mal (valorar lo que no tiene precio asignado, como la conservación del planeta o el sentido de la vida). Por tanto, los mercados necesitan instituciones que los regulen. que canalicen su dinamismo generador de riqueza. Tanto más cuanto que nuestra extraordinaria capacidad tccnológica actual pucde acelerar los efectos, tanto positivos como negativos, de los mercados". Lo que Seatule signilica es el lin cle la ilusión neoliberal de un planeta autogestionado por los mercados para henefício de los más fuertes, de los más listos y, también. de los más pillos.

"Seattle fue un punto de inflexión en la dinámica de nuestro mundo. Lo que ha cambiado Seattle es que a partir de ahora hay que inlormar, hay que discutir, hay que negociar. No sólo porque es más ético y democrático, sino porque es la única manera. La globalización será democrática, informada y controlada por la gente o no será, deshecha por las resistencias múltiples e intereses incompatibles. No será fácil. Llevará tiempo y obligará a concesiones de todas las partes, a explicaciones reiteradas, a malentendidos recíprocos. Pero puede salir y entonces sí beneficiará a los pobres del mundo y a todos los demás. Pero lo que se acabó es la tiranía del mercado, presentada como ley natural", apunta Castells".

Además de resumen, estas reflexiones de Manuel Castells nos sirven para cvitar cl reduccionismo mercantil en los tratados de libre comercio continental o regional. Si los dieciocho anexos del ALCA y las ocho iniciativas del PPP no son simple letra pequeña, que los gobiernos signatarios no leen, es decir, si son algo serio y seriamente suscrito, podemos decir que se quiere entrar en una seria reforma del neoliberalismo latinoamericano. Estaríamos recuperando, con contenido nacional y regional, el significado de Plan de Nación y Programa de $\mathrm{Na}$ ción, así como de una cconomía concertada, participativa. Algo que en Europa occidental se definió como la "planificación por la saliva", o, en palabras de Jcan Monct: "La nación debe ser consultada".

\section{Un Plan de Nación y un Plan de Región}

El Área de Libre Comercio de las Américas nos recuerda que las Américas son varias y distintas por su origen, sus lenguas, su cultura, sus problemas y sus intereses. Aunque la globalización transciende y arrasa las fronteras, un tratado de libre comercio debe tomar en cuenta las identidades regionales, si desea tener éxilo. Canadá, Estados Unidos y México han formado un área de integración comercial, lo cual no ha sido obstáculo para que el presidente Vicente Fox firmara un tratado de libre comercio con la Unión Europea, en el foro de Davos 2001. Con algunas desavenencias y roces aduaneros, avanza la integración del Mercosur, en vías de firmar acuerdos comerciales con la Unión Europea. Los europeos miran con recelo la firma del ALCA por cuanto vendría a debilitar la afinidad comercial con Mercosur. También se hacen esfuerzos para afianzar la lormación del "bloque nacionalista andino", e n estrecha relación con Mercosur y han dado su acuerdo para formar un bloque latinoamericano en el 2002.

El alca: Libre comercio con Plan de Nación 
En este contexto de bloques regionales, el Plan Puebla Panamá vendría a resucitar cl viejo ideal de una integración centroamericana, hoy resquebrajada. La Prensa Gráfica, en su suplemento El Financiero, recuerda los nombres de hombres visionarios "que creyeron que el destino de Centroamérica estaba mejor garantizado a través de la unión de esfuerzos" (10 de junio de 2001, p. 5b). El dato estadístico del elevado porcentaje de exportaciones e importaciones con los Estados Unidos no debe opacar la necesidad y la urgencia de una integración cle lo que se define por Mesoamérica, para enfrentar juntos los problemas comunes y trascender la óptica mercantilista, que reduce todo a relaciones comerciales, como si de ahí, por rebalse, se resolviera el resto de los problemas. Volveríamos así al fallido esfuerzo de presentar programas y planes de nación que se basan en problemas comunes y propuestas alternativas de solución.

La nación quiere participar. Al leer las ocho iniciativas del PPP, inmediatamente me vino a la memoria el índice de las diecinueve mesas de trabajo, organizadas por más de doscientos profesionales (ad honorem), creando el documento Temas claves para un plan de nación: consulta especializada, que data cle enero de 1999. He aquí el tema de cada mesa de trabajo: Sociedad sin fronteras (CA); Descentralización y reorganización territorial; Exclusión social y pobreza; Ciencia y Tecnología; La modernización del Estado; Ramas estratégicas de desarrollo y reformas sectoriales pendientes; Desarrollo agropecuario y rural; Nueva cultura laboral y empresarial; Profundización de la reforma del sistema judicial; Ética en El Salvador; Reforma fiscal y priorización de la inversión física; Mcdioambiente y desarrollo; Reforma del sistema de salud; Estrategia nacional de vivienda; Educación, cultura y deportes; Mesa multidisciplinaria de Washington (La nación debe participar); Integración centroamericana; Información y comunicaciones para la democracia y el desarrollo". Evitaríamos así la monotonía de los equilibrios macroeconómicos contables, que, en poco o en nada ayudan a resolver este mosaico de problemas tanto nacionales como regionales.

Por esta razón, sintonizo con el comentario de Roberto Rubio en La Prensa Gráfica sobre el Plan Puebla-Panamá. A riesgo de ser acusado o tergiversado por uno y otro lado, Rubio ve, como una nueva vía, los siguientes conceptos: "Desarrollo multidimensional, ordenamiento territorial, políticas sectoriales y ambientales, políticas de Estado y de largo plazo, Banca de desarrollo, desarrollo rural integral, lograr un cambio estructural en la dinámica económica, lograr una mayor participación de la sociedad civil en el desarrollo, nuevas plataformas de concertación y creación de consensos, elevar el nivel de desarrollo humano y social de la población, fortalecer las tradiciones culturales de la región y el respeto de los derechos de los pueblos indígenas, fortalecimiento de fondos federales que apoyen específicamente a las empresas sociales y proyectos productivos desarrollados por los indígenas, creación o fortalecimiento de Fondos de Fomento al Desarrollo Tecnológico, incentivos fiscales y líneas específicas de apoyo a las aclividades agrícolas, manufactureras y del sector turismo, desa- 
rrollar una cultura de protección del ambiente y los recursos naturales, fortalecer la capacidad de gestión de las autoridades locales" (La Prensa Gráfica, 18 de junio de 2001, p. 24)

Como indica Rubio, puede haber lecturas e intereses muy diferentes. Habrá quienes vean en este plan "nuevas oportunidades de inversión y hacer ganancia", de acuerdo con la lógica de los negocios y el mercado. Sabemos que "el mercado no tiene corazón" y que su juego favorece exclusivamente a las minorías privilegiadas que controlan los mercados. Sin duda, entran en juego variados intereses -nacionales, megaempresariales y locales - También se hace presente la postura maniquea del total rechazo, que en nada es solución para la mayoría de estos problemas regionales". Me interesa repetir una frase del citado economista acerca de los objetivos del PPP: "En efecto, éste posee muchos aspectos que son parte de la agenda de aquellos que hemos venido criticando las políticas predominantes, y que bien quisiéramos que tomen en cuenta nuestros dirigentes".

Un temor normal es que los interlocutores inmediatos del Plan Puebla-Panamá son los gobiernos. No empatamos con la ideología teórica y aplicada de algunos de ellos, que no han dado muestras fehacientes de consultar a la sociedad civil - primer anexo del ALCA—, o que han arrinconado aportes valiosos de esta misma sociedad. Existe el peligro real de que estos gobiernos privilegien los megaproyectos de infiaestructura física, que hambrean las multinacionales. Me ha inquietado una frase que aparece bajo la columna: "Compromiso del BID. La forma de entregar el financiamiento podría hacerse directamente a los gobiernos para ejecutar trabajos de infraestructura, o trabajar con empresas multinacionales". Literalmente esa frase no suena bien, si ese va a ser el eje central del plan, aunque Enrique Iglesias agrega que el plan no se centra sólo en la infraestructura física.

Nos queda un tiempo de espera: no se conoce aún el monto total de la inversión, ni los términos del financiamiento. El BID y el BCIE estarían dispuestos a ayudar, y la CEPAL mira bien el plan. "En noventa días deberán presentar proyectos concretos". Ese será un primer momento para evaluar los objetivos y los beneficiarios más reales o más escondidos del acuerdo, cuáles de las ocho iniciativas se privilegian y cuáles se quedan en letra muerta. Para decirlo en otras palabras, si el PPP está al servicio de los anfitriones del ALCA o si cstá verdaderamente dirigido a la integración económica, social y territorial de Mesoamérica.

Resumiendo la idea central de este comentario: si sumamos e integramos las recomendaciones de Carolina Alas a los dieciocho anexos del ALCA y a las ocho iniciativas del PPP, damos un paso adelante hacia programas y planes concrelos de nación, que trascienden la visión mercantilista de nuestros gobiernos, los monótonos equilibrios contables macroeconómicos, que aporten respuestas a los problemas internos estructurales y nos fortalezcan para tener mejor éxito en el océano de la globalización. Por lo menos, aprendemos que algo distinto hay que hacer.

El ALCA: Libre comercio con Plan de Nación 


\section{Notas}

1. Ibisatc, Francisco Javier, El ALCA, un acuerdo entre desacuerdos, a publicarse en Estudios Centroamericanos (ECA).

2. De Beer, P.: Quebec: 332 dirigeants des Amériques décident de lier libre-echange et démocracie. Le Monde, 23 de abril de 2001.

3. Habel, J.: Integration á marche forcée pour les Amériques. Le Monde Diplomatique, 23 de abril de 2001.

4. Valenzuela, J.: Las protestas cercan la cumbre de Quebec. El País, 22 de abril de 2001.

5. Ibidem.

6. Alas, Carolina: El nuevo entorno internacional del comercio, II parte. Boletín Económico y Social, FUSADES, $N^{\circ} 180$, noviembre de 2000.

7. Luttwak, E.: Turbocapitalismo. Quiénes ganan y quiénes pierden con la globalización. Ed. Crítica, Barcelona, 2000.

8. El neoliberalismo en América Latina. Realidad, $\mathrm{N}^{\circ} 54$.

9. Hinkelammert, F.: El huracán de la globalización. Economía informa, $\mathrm{N}^{\circ} 225$, marzo de 1997. Un breve resumen puede hallarse en ECA, 1998, pp. 896-897.

10. Agenda de la Cumbre de Quebec. ввс Mundo, 19 de abril de 2001.

11. Castells. M.: Seattle y el cinismo neoliberal. El País, 3 de enero de 2001. 